\title{
Toxicity of ethylene glycol monomethyl ether on reproduction parameters and histomorphological changes in Wistar rats*
}

(iD) (iD)

Satish T. Panchal ${ }^{1}$, Kauresh D. Vachhrajani ${ }^{2}$, Parimal Solanki ${ }^{3}$ and Piyush Patel ${ }^{4}$

Department of Toxicology, Sun Pharma Advanced Research Co Ltd., Vadodara 390010 Department of Zoology, Maharaja Sayajirao University of Baroda, Vadodara 390002

Citation: Satish, T. P., Kauresh, D. V., Parimal, A. S. and Piyush, P. 2021.Toxicity of Ethylene Glycol Monomethyl Ether on reproduction parameters and histomorphological changes in Wistar Rats. J. Vet. Anim. Sci. 52(1): 65-72. DOI: https://doi.org/10.51966/jvas.2021.52.1.65-72

Received : 09.12.2020

Accepted: 26.12 .2020

Published: 01.01.2021

\section{Abstract}

The aim of this study was to evaluate the toxicity of Ethylene Glycol Monomethyl Ether (EGME) on fertility and early embryonic development to implantation, following oral gavage to Wistar rats. EGME, which is a known testicular toxicant, was administered to male rats for four weeks and to female rats for two weeks prior to mating at dose levels of 20, 40 and $80 \mathrm{mg} / \mathrm{kg}$ orally, once daily. Dosing was continued in males until sacrifice (Day 43) and in females until day six of gestation. Females were sacrificed on Day 15 of gestation and examined for implantation sites, viable fetuses and ovarian corpora lutea. Males were evaluated for sperm parameters as well as organ weight and histopathology of the reproductive tissues. At the end of dosing, the $80 \mathrm{mg} /$ $\mathrm{kg} /$ day males had decreased weight and size of testes and epididymides which correlated with tubular atrophy of the testes and ductal atrophy plus reduced sperm in the epididymides. Testicular changes were less severe in the $40 \mathrm{mg} / \mathrm{kg} /$ day group, comprising Sertoli cell vacuolation, with degeneration and depletion of elongating spermatids and spermatid retention in the testes and lumenal cell debris in the epididymides. No testicular abnormalities were observed in the $20 \mathrm{mg} / \mathrm{kg} /$ day males but cell debris was present in the epididymal lumen. There was a dose related decrease in total sperm count and sperm viability ( $\geq 20 \mathrm{mg} / \mathrm{kg} /$ day) and sperm motility ( $\geq 40 \mathrm{mg} / \mathrm{kg} / \mathrm{day})$. The fertility index in the EGME dosed groups showed a dose-related decline and the time taken for females to conceive was increased.

Key words: EGME, testis, implantation, rats

Ethylene glycol monomethyl ether (EGME) and its acetate ester (MEA) are highly volatile liquids used in paints, lacquers, stains, inks and surface coatings, silk-screen printing,

${ }^{\star}$ Forms part of the PhD thesis of the first author submitted to the MS University of Baroda

Correspondence E-mail: satish.panchal@gmail.com,Ph : 9825319421

1 PhD Scholar, M S University of Vadodara

2. Professor, Department of Zoology and Head (Offg) Department of Environmental Studies, M S University of Baroda

3. Manager, Department of Toxicology, Sun Pharma Advanced Research Co Ltd

4. Pathologist, Department of Toxicology, Sun Pharma Advanced Research Co Ltd

Copyright: (C) 2021 Satish et al. This is an open access article distributed under the terms of the Creative Commons Attribution 4.0 International License (http://creativecommons.org/licenses/by/4.0/), which permits unrestricted use, distribution, and reproduction in any medium, provided the original author and source are credited. 
photographic and photo lithographic processes and as an anti-icing additive in hydraulic fluids and jet fuel (Johanson, 2000; Starek and Szabla, 2008). These compounds may be ingested, inhaled or absorbed into the systemic circulation via the dermal route after contact on the skin (Johanson, 2000). EGME and MEA both are listed under Proposition 65 of the Safe Drinking Water and Toxic Enforcement Act of 1986 as known agents causing developmental toxicity and reproductive toxicity ("Ethylene Glycol Monomethyl Ether I OEHHA," 1989). Reports suggest presence of oligospermia and azoospermia and the odds ratio for lower sperm counts per ejaculate were found increased in workers as compared to those workers who were not exposed to glycol ethers, in a study conducted on shipyard painters occupationally exposed to EGME and MEA (Welch et al., 1988). Other cross-sectional studies were also conducted to relate occupational exposure of EGME and male infertility and showed negative effects of the ethers (Cherry et al., 2008; Multigner et al., 2007). In women, disturbance in the corpus luteum function and inhibition of ovulation as well as disturbance of the menstrual cycle have been observed (Davis et al., 1997; Gold et al., 1995). In this study, we have examined the toxicity of EGME on fertility and early embryonic development to implantation following exposure by oral route in male and female Wistar rats at three different dose levels of EGME.

\section{Material and Methods}

\section{Experimental Protocol:}

The study was carried out as per the guidelines provided by the Committee for the Purpose of Control and Supervision of Experiments on Animals (CPCSEA), Government of India. The project was approved by Institutional Animal Ethics Committee. The test facility is accredited with GLP certificatefrom National GLP Compliance Monitoring Authority, Govt. of India and additionally accredited by AAALAC International for animal ethics. Wistar rats (50 male and 50 female, of $150-250 \mathrm{~g}$ ) were received from Laboratory Animal Resources, Sun Pharma Advanced Research Co. Ltd., Vadodara and were housed in Individually
Ventilated Caging system. Sterilized corncob was provided as bedding material. Controlled environment was maintained (light: $12 \mathrm{~h}$ light/ dark; Temperature: $20-26^{\circ} \mathrm{C}$; $\mathrm{RH}$ : $30-70 \%$ ). Animals were provided with Irradiated Certified rodent diet (Envigo, 2018C) and autoclaved R.O. water ad libitum. Females were virgin. Animals were of $200-300 \mathrm{~g}$ at the initiation of dosing and acclimatized for 7 to 10 days. Veterinary health check and randomization was performed during acclimation period to select 48 males and 48 females for study. Animals were divided into 4 groups with 12 animals/ sex/group. EGME was dissolved in de-ionized water and to a final concentration of $2,4,8 \mathrm{mg} /$ $\mathrm{ml}$; rats were given 20 (G2, low dose), 40 (G3, mid dose) and 80 (G4, high dose) mg EGME per kg per day orally. Dose volume of each animal was calculated based on the recently recorded body weights. Control animals (G1) received de-ionized water. Dosing in females of the respective group was initiated on Day 15 of male dosing. Dosing was continued through the mating period also. Dosing in mated females was continued up to Day 6 of gestation and in males until terminal sacrifice (total 42 days of dosing in males).

\section{Mating Protocol and Justification of Doses and Route Selection:}

On last day of pre-mating dosing (i.e. Day 28 in male and Day 14 in female), males and females from the same group were cohabited (1M:1F) for mating. Mating period was up to 10 days or if the female was found to be mated (presence of vaginal plug or presence of sperms in vaginal smear), whichever was earlier. During the mating period vaginal smears were obtained daily in the morning and examined under the microscope for presence of spermatozoa. Vaginal canal was lavaged with normal saline $(0.9 \%)$ and the contents were delivered onto a glass slide. Smears were observed under a microscope and presence of sperms was considered as positive sign of mating i.e. Day 0 of pregnancy. If any female failed to mate during mating period of 10 days; Day 11 was considered as Day '0' of gestation for that female/s. By exposing to Isoflurane (anesthesia) and CO2 (euthanasia), females were sacrificed on gestation Day 15 and males 
were sacrificed on Day 43 and observed for gross pathology.

The doses were selected on the basis of 2 and 4 week repeated dose oral toxicity study of EGME in rats (Dodo et.al., 2009, Watanabe et.al., 2000). Doses of EGME employed in these studies were $0 / 30 / 100 / 300 \mathrm{mg} / \mathrm{kg} /$ day for females (Dodo et al., 2009) whereas males were dosed for $100 / 200 \mathrm{mg} / \mathrm{kg} /$ day for 2 weeks and $100 \mathrm{mg} / \mathrm{kg} /$ day for 4 weeks (Watanabe et al., 2000).

\section{Chemicals:}

Ethylene Glycol Monomethyl Ether (CAS Number: 109-86-4) was purchased from MP Biomedical, LLC, 29525 Fountain Parkway, Solon, OH 44139, United States.

\section{Body weight, Food consumption and Clinical Sign:}

Males were weighed on Day1 of premating dosing and weekly thereafter, and on day of sacrifice. Female body weights were recorded on Day1 of premating dosing and thereafter weekly until found mated. During pregnancy, body weight was recorded on Days $0,3,7,10$ and on day of sacrifice. Feed weights were recorded weekly during pre-mating dosing and until cohabited for mating and in females on gestation Days $0,3,7,10$ and on the day of sacrifice.

Cage side observations were done, once daily, for all rats during the acclimation period and twice daily for all rats, once before dosing and post-dosing until rats were sacrificed.

\section{Necropsy, Histopathology, Sperm Evaluation and Reproductive Performance/ Parameters:}

Females were sacrificed on Gestation Day (GD) 15 and males were sacrificed on Day 43 of the treatment period using isoflurane (for anesthesia) and CO2 (for euthanasia) and observed for gross pathology. $10 \%$ ammonium sulphide solution was used to identify post implantation loss (early death). Number of live and dead conceptus and number of corpora lutea were recorded. The following tissues were removed, blotted, and weighed: epididymis, kidneys, spleen, thymus, lungs, liver, pituitary, right and left testis, brain, adrenal, ovaries and uterus-cervix. The tissues were then stored in $10 \%$ neutral buffered formalin until processed for sectioning except testes and epididymis, which were preserved in Modified Davidson Fixative for $24 \mathrm{~h}$ and then transferred to $10 \%$ neutral buffered formalin for storage. Sections of testes and left epididymis of animals from all groups were taken at four to six microns and stained with periodic acid schiff (PAS) and haematoxylin-eosin (H\&E). Measurement of sperm motility, viability, total sperm count and morphology from all animals was performed manually (Hood, 2005) as per below procedures. Cauda of right epididymis was used from all animals.

\section{Sperm motility:}

A small piece of right cauda was collected in $5 \mathrm{ml}$ of $0.9 \%$ normal saline in petriplate kept at room temperature $\left(23-28{ }^{\circ} \mathrm{C}\right)$ Sperms were allowed to ooze out in saline for approximately 5-10 minutes. A small drop of saline contained semen from junction of cauda was taken with pipette and loaded the haemocytometer chamber. WBC counting squares in haemocytometer were observed under $10 x$ objective and counting of non-motile sperms followed by counting of total sperms was done. Percentage motility was determined as: Motile spermatozoa $=$ Total spermatozoa count - Non-motile spermatozoa

Motility (\%) = Motile spermatozoa/Total Spermatozoa X 100

\section{Total sperm count:}

The remaining part of cauda was weighed $(\mathrm{mg})$ and crushed with BP blade and forceps in $5 \mathrm{ml}$ of normal saline in a petriplate kept at room temperature $\left(22-26^{\circ} \mathrm{C}\right)$ Sperms were allowed to ooze out into saline for approximately 5-10 minutes. A homogenate was obtained by swirl action of contents in petriplate to get maximum free spermatozoa. The sample was sucked in WBC diluting pipette up to the 0.5 marks and then normal saline up to 11 marks on WBC pipette (20 times dilution). It was 
mixed well by rotating the pipette between two palms. First few drops were discarded and then haemocytometer was charged with the diluted sample. Total spermatozoa were counted from WBC counting squares under $10 \mathrm{X}$ objective. Total sperm count was determined as follows:

Total sperm count $=\{(\mathrm{N} \times 20 \times 1000 \times$ Dilution Volume)/ 0.4$\}$ in weighed cauda

$\mathrm{N}=$ Total number of spermatozoa in four WBC counting squares

Total sperm count was expressed as number of spermatozoa per gram of cauda.

\section{Sperm morphology:}

Sperm morphology was determined from sample prepared as above (followed for Total sperm count). $100 \mu \mathrm{L}$ of the sample was added in $500 \mu \mathrm{L}$ of $10 \%$ neutral buffered formalin in micro centrifuge tube. After proper mixing, smear was prepared, in duplicates, on clean and grease free slides. Slides of each animal were identified with animal ID number followed by set A or set B. First set of slide (i.e. A) was stained for evaluation. Other set (i.e. B) was not used, as slide from set $A$ was satisfactory. Slide was stained with $1 \%$ eosin for 30 minutes, air dried and observed under microscope (10-40X objective) on next day after mounting with DPX mountant and coverslip. At least 200 spermatozoa was observed for morphology and any abnormality such as tailless sperm, headless sperm, no hook head sperm, neck abnormality, double head, double tail, coiled tail, bent tail were recorded. At the time of archival slides will be identified with study number and animal ID. Percentage of abnormal spermatozoa was determined as below:

Abnormal spermatozoa $(\%)=($ Total no. of abnormal spermatozoa/Total sperm observed) X 100

\section{Sperm viability:}

Sperm viability was determined from sample prepared as above (Sperm Motility). About 2- 5 minutes after cauda collection, $5 \mu \mathrm{L}$ of the sample was taken on glass slide and mixed with $5 \mu \mathrm{L}$ of normal saline and $5 \mu \mathrm{L}$ of $0.5 \%$
Eosin (in normal saline) each by swirling action using micropipette tip. Slide was observed under microscope at $40 \mathrm{X}$ for counting viable and non-viable sperms. Viable sperms remain colourless while nonviable sperms remain red colour. Total 200 sperms (viable plus nonviable) were counted. Viability was calculated as below.

Sperm Viability $(\%)=($ Viable Sperm $/$ Total Sperm) X 100

Male mating index, male fertility index, females mating index, female fertility index, implantation index, pre-implantation loss, post-implantation loss, fecundity index and live/dead conceptus were calculated. Formulas for calculations are as follows:

Male mating index $(\%)=($ No. of males with confirmed mating/No. of males cohabitated) $\mathrm{x}$ 100

Male fertility index (\%) = (No. of males impregnatingafemale/No. of males cohabitated) $\mathrm{x} 100$

Female Mating Index (\%) = (No. of females mated/No. of cohabitated females) $\times 100$

Female Fertility Index (\%) = (No. of pregnant females/No. of females with cohabitated) x 100

Implantation Index $(\%)=($ No. of implants $/$ No. of corpora lutea) x 100

Pre - Implantation loss (\%) $=$ (No. of implants) No. of corpora lutea - No. of implants/No. of corpora lutea) x 100

Post - Implantation loss (\%) = (No. of implants) No. of implants - No. of viable fetuses $/$ No. of implants) $\times 100$

Fecundity Index $(\%)=$ (No. of pregnant females) No. of females with confirmed mating) $\times 100$

\section{Statistical analysis:}

One-way analysis of variance $(p<0.05)$ and Dunnett's test was done.

\section{Results and Discussion}

\section{Body Weight and Food Consumption:}

Compared with controls $(11.58 \%$ 
gain at week 6), treatment related significant decrease in percent body weight changes was observed in G4 group males (1.46\% gain at week 6) throughout the experimental period. Compared with control (22.1g/day/ animal at week 2 and 19.29g/day/animal at week 4), treatment related statistically significant decrease was observed in the feed consumption in Week 2 (16.68g/day/animal) and Week 4 (14.72g/day/animal) for G4 males, while females from G2 (10.4g/day/animal), G3 (10.63g/day/animal) and G4 (10.28g/day/ animal) groups showed treatment related significant decrease in feed consumption in Week 2 in comparisons to control (12.44g/day/ animal).

\section{Necropsy and Histopathology}

\section{Organ Weights:}

In G4 males, statistically significant decrease in absolute and relative weights were seen in epididymides and testes (Table 1).

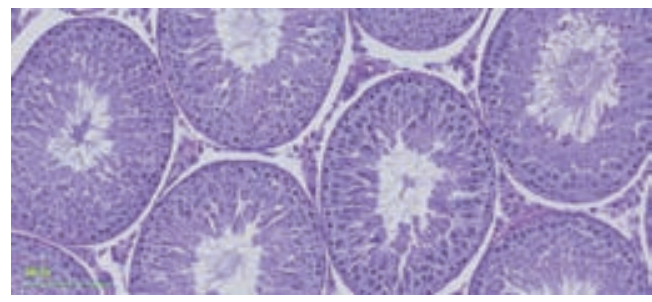

Fig 1a Control Group - 102 - 20x Normal Testis

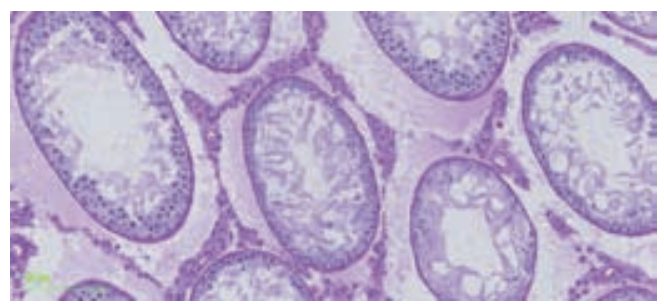

Fig 1b High dose - 20x

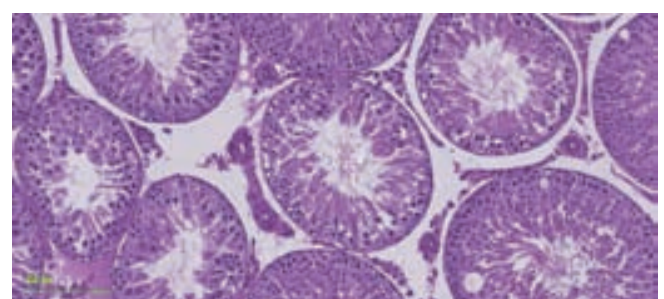

Fig 2 - 20x Sertoli Cell Vacuolation and Partial Germ Cell Loss

\section{Macroscopic Observation:}

In G4 males, gross findings included small testes (11/12), epididymides (8/12), adrenals (4/12) and thymus (2/12) which correlated with decreased organ weights.

\section{Microscopic Observation:}

Testes: Tubular degeneration/atrophy was present in the testes of all rats in G4 (12/12) and in most rats $(7 / 12)$ rats from G3. The lesion was severe in G4 and minimal to slight in G3. No abnormalities were identified in the testes of G2. In G4 rats, most tubules had depletion of elongating spermatids, round spermatids and pachytene spermatocytes, leaving tubules lined by Sertoli cells, spermatogonia and prepachytene spermatocytes (Fig 1b). Partial germ cell depletion and actively degenerating (apoptotic) germ cells were observed in some tubules with disorganisation of the remaining germ cells. The affected tubules were reduced in diameter, resulting in expansion of the

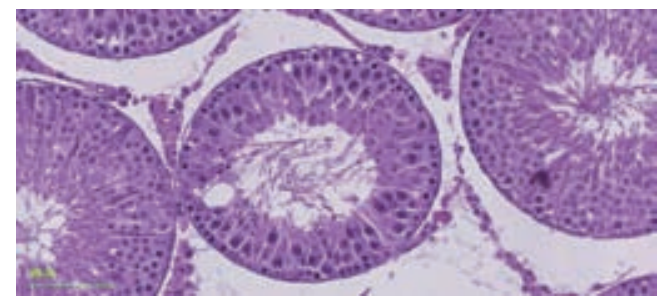

Fig 3 Mid Dose - 30x Spermatid Retention

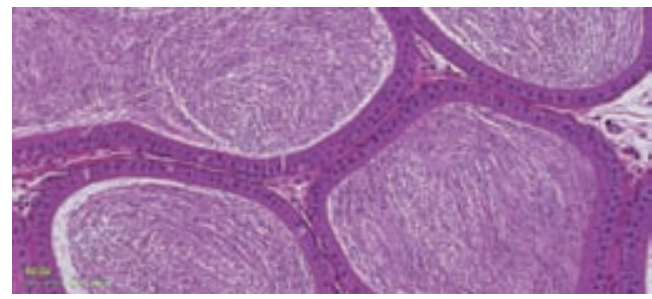

Fig 4a Control - 20x caput

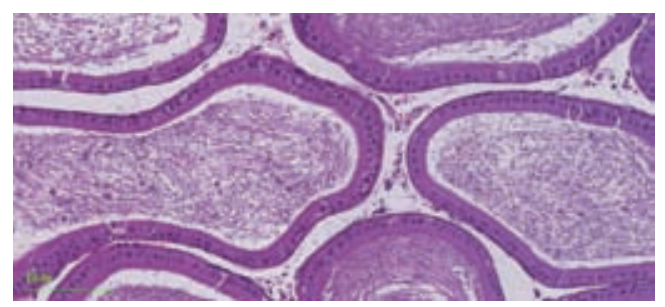

Fig 4b Low Dose -20x Cell debris in caput 
Table 1: Major findings on male and female treated with EGME

\begin{tabular}{|c|c|c|c|c|c|}
\hline \multicolumn{2}{|r|}{ EXPOSURE LEVEL (mg/kg/day) } & Deionized water & 20 (G2) & 40 (G3) & 80 (G4) \\
\hline \multirow{11}{*}{ Male } & Total Sperm Count $\left(\mathrm{X} 10^{6}\right) / \mathrm{gm}$ of Cauda & $644.85 \pm 138.095$ & $549.89 \pm 200.325$ & $304.46 \pm 137.366$ * & $104.02 \pm 68.261$ * \\
\hline & Sperm Motility $(\%)$ & $76.24 \pm 4.845$ & $51.69 \pm 17.703$ & $50.65 \pm 14.585^{*}$ & $9.58 \pm 9.356$ * \\
\hline & Sperm Viability (\%) & $68.92 \pm 5.961$ & $51.71 \pm 23.506$ & $48.64 \pm 21.620$ & $27.35 \pm 15.202$ * \\
\hline & Sperm Abnormality (\%) & $2.71 \pm 0.450$ & $3.00 \pm 0.477^{*}$ & $3.13 \pm 0.528^{*}$ & $3.04 \pm 0.450^{*}$ \\
\hline & Left epididymis (Weight, g) & $0.7641 \pm 0.07441$ & $0.7549 \pm 0.11608$ & $0.6977 \pm 0.07539$ & $0.5109 \pm 0.06829$ * \\
\hline & Right epididymis (Weight, g) & $0.73623 \pm 0.087157$ & $0.71480 \pm 0.083899$ & $0.67494 \pm 0.058589$ & $0.53153 \pm 0.113885^{*}$ \\
\hline & Testes (Weight, $\mathbf{g}$ ) & $4.07638 \pm 0.351035$ & $3.87877 \pm 0.500557$ & $3.94159 \pm 0.379449$ & $2.01477 \pm 0.401314$ * \\
\hline & Left epididymis (\%) & $0.17237 \pm 0.019665$ & $0.17692 \pm 0.024427$ & $0.15914 \pm 0.019426$ & $0.12614 \pm 0.012900$ * \\
\hline & Right epididymis (\%) & $0.16633 \pm 0.022686$ & $0.16751 \pm 0.015280$ & $0.15438 \pm 0.020742$ & $0.13233 \pm 0.031785$ * \\
\hline & Testes (\%) & $0.92103 \pm 0.115062$ & $0.90775 \pm 0.084522$ & $0.90275 \pm 0.132032$ & $0.49388 \pm 0.066198$ * \\
\hline & Fertility Index (\%) & $11 / 12(91.7 \%)$ & $8 / 12(66.6 .0 \%)^{*}$ & $7 / 12(58.3 \%)^{*}$ & $3 / 12(25.0 \%)^{*}$ \\
\hline \multirow{6}{*}{ Female } & Time to Conception (days) & 1.9 & 5.4 & 2.9 & 4.7 \\
\hline & $\begin{array}{l}\text { Total Corpora Lutea (Mean CL) } \\
\text { from pregnant females (nos.) }\end{array}$ & $148(13)$ & $103(12)$ & $85(13)$ & $33(11)$ \\
\hline & $\begin{array}{c}\text { Live Foetus } \\
\text { (Mean Live Foetus/litter) (nos.) }\end{array}$ & $98(8.9)$ & $79(9.9)$ & $36(5.1 *)$ & $14\left(4.7^{*}\right)$ \\
\hline & Total Implantation Sites (nos.), (\%) & $110(75.90 \%)$ & $86(82.23 \%)$ & $57(67.85 \%)$ & $18(49.76 \%)$ \\
\hline & Mean Implantation Sites (nos.) & 9.2 & 8.6 & 5.1 & $1.5^{*}$ \\
\hline & \% Implantation Loss: Pre and post & 24.10 and 19.25 & 17.77 and 7.26 & 32.15 and 27.08 & 50.24 and 27.35 \\
\hline
\end{tabular}

Note: $n=12,{ }^{*}$ : significantly different from control group $(p<0.05)$

interstitial space but there was no apparent increase in the number or size of Leydig cells. In G3, fewer testes were affected (7/12), and the severity of the changes was much less (predominantlyminimal). Only asmall proportion of tubules were affected, and the changes were characterised by focal Sertoli cell vacuolation, degeneration and depletion of elongating spermatids (Fig 2) and spermatid retention (Fig. 3). In one rat (graded slight severity) the germ cell degeneration and depletion affected spermatids and spermatocytes and was present in more tubules than the remainder of the group.

Epididymides: In G4, most epididymides had severely reduced, or absent sperm content as well as the presence of cell debris, that had originated from the testes. In most cases, epididymides but had normal appearing testes. This is explained by the fact that epididymal cell debris is generally considered a more sensitive indicator of testicular germ cell sloughing than testicular histopathology. In G2, 5/12 rats had epididymal cell debris but had normal appearing testes.

Histopathological changes in the testes are consistent with the known effects of EGME on spermatogenesis. EGME is known to target the spermatocyte within $24 \mathrm{~h}$ of dosing (Foster et al., 1984, Chapin et al., 1985), but with continued dosing, progressive maturation depletion and/or degeneration of subsequent cell types (round and elongating spermatids) were observed.

As per earlier report, EGME causes its maximal effect on fertility parameters after at least 6 weeks of dosing (Anderson et al., 1987). Similarly, in current study, in male rats dosed only for 28 days before mating, the changes in fertility parameters were evident. Males necropsied after 42 days of dosing showed severe testicular toxicity and decreased sperm parameters in the high dose group and less severe changes down to the low dose group. The severity of the testicular toxicity in the high dose group reflects the effects of the long duration dosing, which resulted in an end 
stage lesion comprising depletion of most germ cells except prepachytene spermatocytes and spermatogonia, as well as severe depletion of epididymal sperm.

\section{Sperm Evaluation and Reproductive Performance/Parameters:}

Fertility index (\%) in the EGME dosed groups declined compared to the vehicle control group (G1), and more time (days) was required for females to conceive. Statistically significant $(p<0.05)$ decreases were observed in the number of corpora lutea and \% implantation sites in G4 group females. Dose related, statistically significant $(p<0.05)$ decreases were observed in total sperm count, viability and sperm motility in G3 and G4 males, while in G2 males, a significant $(p<0.05)$ decrease in sperm motility was observed. Sperm abnormality was seen more in EGME dosed groups as compare to the control males. The presence of sperm changes in EGME treated groups correlates with the histopathological abnormalities in the testes and epididymides.

In this study, the low dose was expected to be a NOEL, however, there was a slight diminution in total sperm count, sperm motility and sperm viability and an increase in abnormal sperm. Although not statistically significant, they correlated with a significant decrease in fertility index for this group and are considered to be related to EGME treatment. Histologically, there were no detectable changes in the testes although there was an increased incidence of minimal cell debris in the epididymis, indicating some germ cell sloughing from the testis.

In the mid dose group (40mg/kg/day), there were statistically significant $(p<0.05)$ decreases in total sperm count, sperm motility and in the number of abnormal sperm. These abnormalities were consistent with microscopic evidence of germ cell depletion and degeneration in the testis, which mostly affected the spermatid population, as well as sloughed germ cells (luminal cell debris) in the epididymis. The decreased sperm parameters and histopathological changes correlated with a significantly decreased fertility index in this group. These effects in the high dose group $(80 \mathrm{mg} / \mathrm{kg} /$ day $)$ were more marked than those seen in the mid dose group. All animals in the group showed severe tubular degeneration/ atrophy in the testes and reduced sperm with ductal atrophy in the epididymides. The findings correlated with marked decreases in total sperm count, sperm motility and viability and increased abnormal sperm. The fertility index in this group was only $25 \%$. In addition, the time taken for females to conceive was increased.

In this study females and males were dosed and therefore the possibility exists that the affected fertility parameters (fertility index, time to conception, number of corpora lutea, number of live foetuses and number of implantation sites) could be the result of female mediated toxicity rather than male-mediated toxicity, or could be a mixture of both. However, based on the results of previous studies (Davis et al., 1997, Dodo et al., 2009), female reproductive toxicity of EGME was only seen at doses $\geq 100$ $\mathrm{mg} / \mathrm{kg} / \mathrm{day}$. In those studies, effects included suppression of cyclicity and hypertrophy of the corpora lutea. Contrastingly, in the current study, females were dosed at $<100 \mathrm{mg} / \mathrm{kg} /$ day, so the changes in fertility parameters are considered to be male-mediated. The EGMErelated changes in fertility parameters included a dose-related decrease in the number of corpora lutea, live foetuses, and implantation sites. Most of the implantation loss might be due to preimplanation failure, which is consistent with the decreased numbers and motility of sperm. The small increase in post implantation loss was not dose related and is considered incidental. This is consistent with the study reported by Anderson et al., (1987).

\section{Acknowledgement}

The authors would like to thank Dianne Creasy for helpful assistance during its preparation.

\section{References}

Anderson, D., Brinkworth, M.H., Jenkinson, P.C., Clode, S.A., Creasy, D.M., Gangolli, S.D. (1987). Effect of ethylene glycol monomethyl ether on spermatogenesis, dominant lethality, and F1 abnormalities in the rat and the mouse after 
treatment of F0 males. Teratogenesis, Carcinogenesis, and Mutagenesis, 7(2):141-158.

Chapin, R.E., Dutton, S.L., Ross, M.D., Lamb, J.C. 1985. Effects of ethylene glycol monomethyl ether (EGME) on mating performance and epididymal sperm parameters in F344 rats. Fundam. Appl. Toxicol., 5: 182-189. https://doi.org/10. 1016/02720590(85)90063-6

Cherry, N., Moore, H., McNamee, R., Pacey, A., Burgess, G., Clyma, J.-A., Dippnall, M., Baillie, H., Povey, A., participating centres of Chaps-UK. 2008. Occupation and male infertility: glycol ethers and other exposures. Occup. Environ. Med. 65, 708-714. https://doi.org/10.1136/ oem.2007.035824

Dodo, T., Taketa,Y., Sugiyama,M., Inomata,A., Sonoda,J., Okuda,Y., Mineshima,H., Hosokawa,S., Aoki , T. 2009. Collaboration work on evaluation of ovarian toxicity 11) Two- or four-week repeated-dose studies and fertility study of ethylene glycol monmethyl ether in female rats. J. Toxicol. Sci., 34: Suppl. 1: SP 121-SP128.

\section{Ethylene Glycol Monoethyl Ether I OEHHA} [WWW Document].1989. URL https://oehha.ca.gov/proposition-65/ chemicals/ethylene-glycol-monoethylether (accessed5.3.19).

Foster, P.M., Creasy, D.M., Foster, J.R., Gray, T.J., 1984. Testicular toxicity produced by ethylene glycol monomethyl and monoethyl ethers in the rat. Environ.

Gold,E.B., Eskenazi, B., Hammond, S.K., Lasley, B.L., Samuels, S.J., O’Neill Rasor, M., Health Perspect.57:207-217. https:// doi.org/10.1289/ehp.8457207
Hines, C.J., Overstreet, J.W., Schenker, M.B. 1995. Prospectively assessed menstrual cycle characteristics in female wafer-fabrication and nonfabrication semiconductor employees. Am. J. Ind. Med., 28: 799-815.

Hood, R. D., and Hood, R. D. 2005. Developmental and Reproductive Toxicology: A Practical Approach. Page: 444-458. CRC Press. Retrieved from https://books.google.co.in/ books?id=8X7LBQAAQBAJ,

Johanson, G., 2000. Toxicity Review of Ethylene Glycol Monomethyl Ether and its Acetate Ester. Crit. Rev. Toxicol., 30: 307-345. https://doi. org/10.1080/10408440091159220

Multigner, L., Ben Brik, E., Arnaud, I., Haguenoer, J.M., Jouannet, P., Auger, J., Eustache, F. 2007. Glycol ethers and semen quality: a cross-sectional study among male workers in the Paris Municipality. Occup. Environ. Med., 64: 467-473. https://doi.org/10.1136/ oem.2005.023952

Watanabe, A., Nakano, Y., Endo, T., Sato, N., Kai, K., Shiraiwa, K. 2000. Collaborative work to evaluate toxicity on male reproductive organs by repeated dose studies in rats 27). Repeated toxicity study on ethylene glycol monomethyl ether for 2 and 4 weeks to detect effects on male reproductive organs in rats. J. Toxicol. Sci., 25: 259-266.

Welch, L.S., Schrader, S.M., Turner, T.W., Cullen, M.R. 1988. Effects of exposure to ethylene glycol ethers on shipyard painters: II. Male reproduction. Am. J. Ind. Med., 14:509-526. 